ARTICLE

https://doi.org/10.1038/s41467-022-28070-9

\title{
Single-component color-tunable circularly polarized organic afterglow through chiral clusterization
}

Hui $\mathrm{Li}^{1}$, Jie Gu${ }^{1}$, Zijie Wang ${ }^{1}$, Juan Wang ${ }^{1}$, Fei He${ }^{1}$, Ping $\mathrm{Li}^{1}, \mathrm{Ye}$ Tao (i] ${ }^{1 凶}$, Huanhuan $\mathrm{Li}^{1}$, Gaozhan Xie ${ }^{1}$, Wei Huang (i) ${ }^{1,2 \otimes}$, Chao Zheng ${ }^{1} \&$ Runfeng Chen (1) ${ }^{1 \times}$

Circularly polarized organic afterglow (CPOA) with both long-lived room-temperature phosphorescence (RTP) and circularly polarized luminescence (CPL) is currently attracting great interest, but the development of multicolor-tunable CPOA in a single-component material remains a formidable challenge. Here, we report an efficient strategy to achieve multicolor CPOA molecules through chiral clusterization by implanting chirality center into non-conjugated organic cluster. Owing to excitation-dependent emission of clusters, highly efficient and significantly tuned CPOA emissions from blue to yellowish-green with dissymmetry factor over $2.3 \times 10^{-3}$ and lifetime up to $587 \mathrm{~ms}$ are observed under different excitation wavelengths. With the distinguished color-tunable CPOA, the multicolor CPL displays and visual RTP detection of ultraviolent light wavelength are successfully constructed. These results not only provide a new paradigm for realization of multicolor-tunable CPOA materials in single-component molecular systems, but also offer new opportunities for expanding the applicability of CPL and RTP materials for diversified applications.

\footnotetext{
${ }^{1}$ State Key Laboratory of Organic Electronics and Information Displays \& Institute of Advanced Materials (IAM), Nanjing University of Posts \& Telecommunications, 9 Wenyuan Road, Nanjing 210023, China. ${ }^{2}$ Institute of Flexible Electronics (IFE), Northwestern Polytechnical University, Xi'an 710072

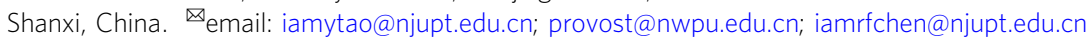


C ircularly polarized luminescent (CPL) materials with efficient emission dissymmetry factor $\left(g_{\text {lum }}\right)$ have received widespread attention recently on account of their attractive potential applications in diversified optoelectronic applications ${ }^{1-6}$. Especially, the pleochroism of chiral luminescent materials can empower multiplexing confidentiality for multilevel data encoding and encryption ${ }^{7}$; the multicolor CPL materials with stimuli-response attributes are idea emissive mediums for brilliant liquid crystal and stereoscopic display ${ }^{8}$; high sensitivity and spatial resolution luminescence sensors for biological analytes and object identification could also be gifted by employing polychromatic chiral molecules as optical marker recognition ${ }^{9}$. In these applications, multicolor-tunable CPL emissions are highly desired, but can be only realized in multicomponent material systems on the basis of various design methods including multicomponent supramolecular assembly 10,11 , chemical additive induced excited state modulation ${ }^{12-14}$ and solvation effect enabled structural color change ${ }^{15}$, etc. Nevertheless, it remains a daunting challenge to develop efficient multicolor-tunable CPL emission from a single-component material system with the capability to dynamically and selectively respond to the changes of physical parameters of external stimuli such as electrical and magnetic fields, pressure, and optical input ${ }^{16}$.

Circularly polarized organic afterglow (CPOA) with both the ultralong-lived triplet excited state for room-temperature phosphorescence (RTP) and efficient CPL features is an amazing type of luminance that has emerged as a hot research topic recently because of the distinguished photophysical phenomena in various remarkable applications ${ }^{17-20}$. Over the past few years, a set of design approaches including multicomponent host-guest ${ }^{21}$, ionic co-crystal ${ }^{22}$, and chiral chain engineering ${ }^{23}$ have been proposed to promote the intersystem cross (ISC) and suppress nonradiative decay of triplet excitons, and to boost efficient chirality transfer from chiral groups to chromophores simultaneously for the realization of CPOA materials with lifetime over $100 \mathrm{~ms}$. Nonetheless, the afterglow emission colors of CPOA materials are limited to fixed emission colors, mostly in yellow or yellowishgreen under ambient conditions ${ }^{19,21-24}$; it is rather difficult to develop multicolor-tunable CPOA materials due to the complicated chirality transfer between the chiral unit and the RTP emission group for CPL as well as the inefficient RTP color tuning for multicolor emission, especially in a molecule. To the best of our knowledge, the CPOA material showing multicolortunable CPL emission from a single-component molecule system has not yet been reported.

Here, we propose an efficient strategy to realize color-tunable CPOA from a single-component non-conjugated molecule by chiral clusterization. Specially, the non-conjugate cluster shows size-dependent clusterization-triggered emission (CTE) via through-space conjugation (TSC) for efficient excitationdependent color-tunable organic afterglow emission due to the presence of varied luminescent centers with different cluster sizes (Fig. 1a) ${ }^{25,26}$. Inspired by the flexible chiral chain engineering to realize CPOA in a single-component purely organic small molecule, we suspect that the color-tunable CPOA can be also designed by implanting the chiral chain into a single-component cluster crystal. Indeed, through a large number of in-plane and interlayer molecular interactions and the dense in-plane and interlayer non-covalent bond network to support the formation of varied emission species and suppression of the non-radiative transition of excitons (Fig. 1b, c) ${ }^{27-29}$, efficient color-tunable CPOA with emission peaks from 470 to $530 \mathrm{~nm}$, lifetimes up to $587 \mathrm{~ms}$, and the $\left|\mathrm{g}_{\text {lum }}\right|$ over $2.3 \times 10^{-3}$ were observed upon tuning the excitation wavelength of ultraviolet (UV) light. Based on the spectacular feature of the color-tunable CPOA molecules, multicolor CPL displays and visual RTP detection of UV light were successfully established. These findings in purposefully constructing multicolor CPOA with the on-demand color-tuning ability through the elaborate molecular design by chiral clusterization would provide a promising platform for the exploration of single-component smart CPL and RTP materials.

\section{Results}

Material design and synthesis. To confirm our hypothesis, we designed and prepared a pair of enantiomers composed of chiral trans-1,2-diamidocyclohexane core and two succinic acid arms (Fig. 1d ${ }^{30}$. In this motif, chiral trans-1,2-diamidocyclohexane can not only employ as a chiral center but also can facilitate the intersystem crossing (ISC) promoted by the nitrogen (N) atoms to accelerate the production of triplet excitons. Meanwhile, bearing the carbonyls $(\mathrm{C}=\mathrm{O})$ and intra/intermolecular bonding sites, the succinic acid unit can remarkably boost the ISC process and more importantly ${ }^{31-33}$, establish strong non-covalent bond interactions between different molecules to form interlocked molecular system for the suppression of non-radiative decay and formation of varied clusters with different sizes. In addition, benefitting from the multiple heteroatoms and varied clusters, TSC originated from the orbital overlap of $\left(n, \pi^{*}\right)$ and $\left(\pi, \pi^{*}\right)$ can be effectively conferred to endow tunable triplet energy levels in different clusters ${ }^{34}$. As a proof of concept, with the molecular design strategy, the enantiomers of $(\mathrm{R}, \mathrm{R})-\mathrm{DAACH}$, and $(\mathrm{S}, \mathrm{S})$ DAACH were facilely synthesized by a one-step amidation reaction between trans- $(1 \mathrm{R}, 2 \mathrm{R}) /(1 \mathrm{~S}, 2 \mathrm{~S})$-diamidocyclohexane and succinic anhydride. This one-step reaction enables high yields up to $88 \%$ and the resultant molecules were systematically characterized by nuclear magnetic resonance (NMR) spectra, high-resolution mass spectra (HRMS), matrix-assisted laser desorption/ionization time of flight mass spectrometer (MALDITOF MS) and thermal physical properties (Supplementary Figs. 1-13).

Photophysical properties. Expectantly, excitation-dependent color-tunable CPOA is successfully realized in singlecomponent chiral cluster crystal of (R, R)-DAACH and (S, S)$\mathrm{DAACH}$, showing long-lived emissions ranging from blue to yellowish-green with moderate $\left|g_{\text {lum }}\right|$ over $2.3 \times 10^{-3}$ under the excitation by 240,340 , and $360 \mathrm{~nm}$, respectively (Fig. $2 \mathrm{a}, \mathrm{b}$ and Supplementary Fig. 14). The chiral characteristics were also observed, when $(\mathrm{R}, \mathrm{R})-\mathrm{DAACH}$ and $(\mathrm{S}, \mathrm{S})-\mathrm{DAACH}$ powders were dispersed $(50 \mathrm{wt} \%)$ in potassium bromide slice (Supplementary Fig. 15). The almost mirror circular dichroism signals of (S, S)-DAACH and (R, R)-DAACH ethanol solutions with strong signals at $\sim 205 \mathrm{~nm}$ further confirm the successful introduction of chirality in these materials (Supplementary Fig. 16). The excitation-phosphorescence spectra of (R, R)-DAACH and (S, S)$\mathrm{DAACH}$ powders demonstrated that with varying the excitation wavelength tuning from 200 to $450 \mathrm{~nm}$ (Fig. 2a, bottom panel and Supplementary Fig. 17), the gradually red-shifted CPOA with main emission peaks tuning from 470 to $530 \mathrm{~nm}$ and decreased luminescent intensities were achieved, which were also manifested by the resolved CPOA spectra (Supplementary Figs. 18-19) and corresponding Commission International de l'Eclairage (CIE) (Fig. 2c) upon excitation by a different wavelength ranging from 220 to $360 \mathrm{~nm}$ with a step of $20 \mathrm{~nm}$; also, a nearly linear relationship between the variations in CIE coordinates and excitation wavelength was obtained, indicating the great feasibility of this color-tunable CPOA in serving as RTP sensing chart for detecting UV light.

To further investigate the influence of the excitation wavelength on the color-tunable CPOA properties, the time-resolved emission spectra were performed ${ }^{35}$. As shown in Fig. $2 \mathrm{~d}$ and 
a

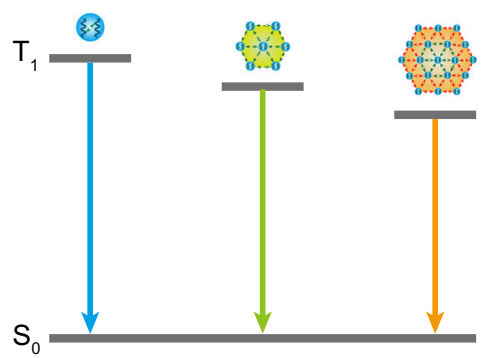

b

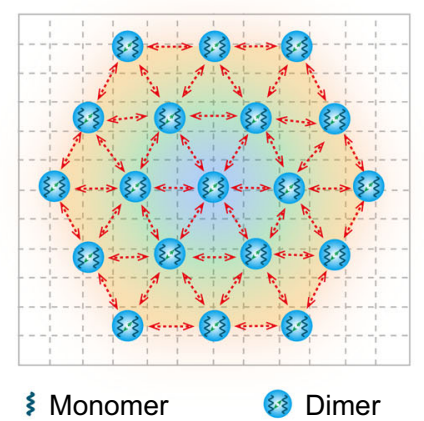

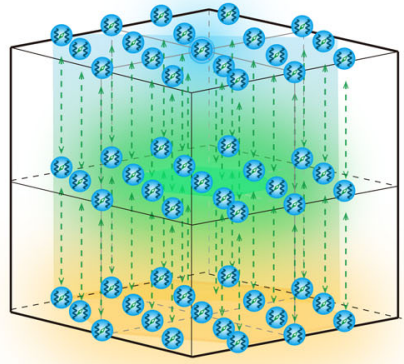

$\leftrightarrow \leftrightarrow \leftrightarrow$ Intermolecular interaction

C

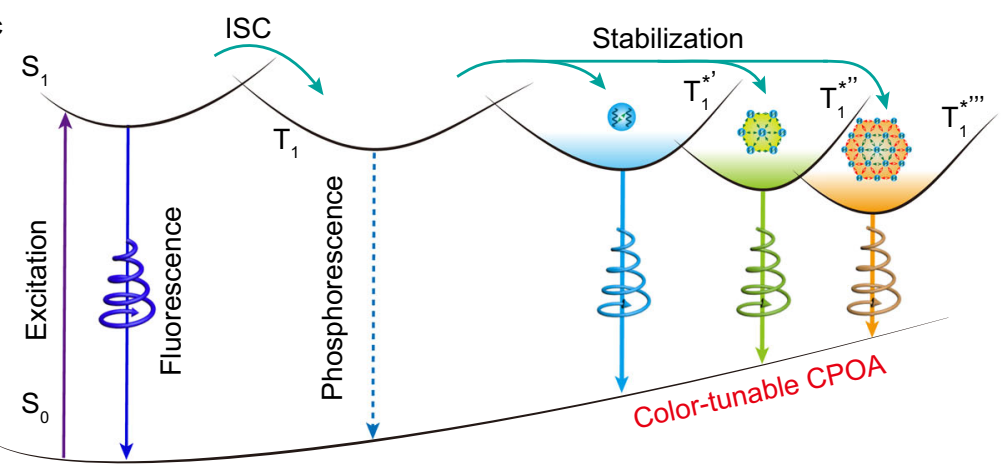

d

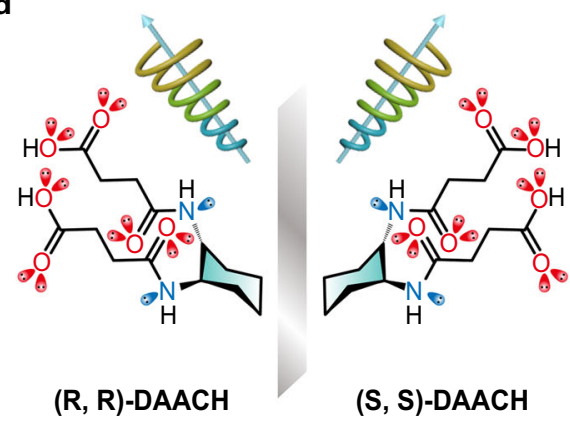

Fig. 1 Molecular design of single-component color-tunable CPOA. a Proposed mechanism of color-tunable phosphorescence emission from different cluster sizes showing tunable excited energy levels. b Top and lateral views of molecular packing in the chiral cluster crystal. The dotted arrows represent intra/intermolecular interactions. The blue ball and the hexagons with different background colors ranging from blue to orange represent size-dependent CTE centers showing color-tunable CPOA. c, d Proposed mechanism (c) and molecular design (d) of color-tunable CPOA from a single-component chiral cluster crystal. So is the ground state. $\mathrm{S}_{1}$ and $\mathrm{T}_{1}$ represent the lowest singlet and triplet excited states. $\mathrm{T}_{1}{ }^{\prime \prime}, \mathrm{T}_{1}{ }^{* \prime \prime}$, and $\mathrm{T}_{1}{ }^{{ }^{* \prime \prime}}$ are the stabilized triplet excited states originated from varied emission species showing different cluster sizes, respectively.

Supplementary Figs. 20-21, both (R, R)-DAACH and (S, S)DAACH powders indicated excitation-dependent CPOA decay profiles, displaying tunable ultralong lifetimes spanning from 130 to $460 \mathrm{~ms}$ for $(\mathrm{R}, \mathrm{R})-\mathrm{DAACH}$ and 324 to $587 \mathrm{~ms}$ for $(\mathrm{S}, \mathrm{S})$ DAACH powders upon excitation turned from 220 to $360 \mathrm{~nm}$, respectively. These distinct variations in lifetime further confirmed the presence of disparate emissive species ${ }^{26}$. Meanwhile, the transient emission decay images of (R, R)-DAACH and (S, S)$\mathrm{DAACH}$ powders verified that this color-tunable emission is quite stable over time under the excitation by different wavelengths (Fig. 2e and Supplementary Fig. 22). The changes in CPOA intensities as a function of irradiation time and intensity showed that excellent CPOA properties could be stimulated under the short excitation duration of $0.1 \mathrm{~s}$ and low excitation power density of $0.11 \mathrm{~mW} / \mathrm{cm}^{2}$ (Fig. $2 \mathrm{f}, \mathrm{g}$ and Supplementary Figs. 23-25). Possibly due to the relatively slow and weak intersystem crossing rates, it needs considerable time to achieve the stable state of triplet excitons for afterglow emission, resulting in the slightly enhanced RTP with the increase of photoirradiation time (Fig. 2f and Supplementary Fig. 23). Moreover, with the excitation by $240 \mathrm{~nm}$, CPOA revealed higher intensity compared to that of the excitation wavelength of $360 \mathrm{~nm}$. As demonstrated in Fig. $2 \mathrm{~h}$, with the excitation by UV light from 200 to $330 \mathrm{~nm}$ the sky-blue CPOA emissions from (R, R)-DAACH powder were much higher than these of yellowish-green; in contrast, when they were motivated by UV light from 330 to $450 \mathrm{~nm}$, the yellowish-green CPOA became the dominated emission; these color changes upon tuning the excitation wavelength further confirmed the presence of varied emissive species for the color-tunable CPOA emission.
To gain a deeper insight into these distinct photophysical properties, the steady-state photoluminescence (SSPL) spectra were further monitored. The SSPL of $(\mathrm{R}, \mathrm{R})-\mathrm{DAACH}$ is also excitation-dependent, exhibiting slight hypochromatic shift for varied colors from sky-blue to blue and tunable quantum yields when the excitation wavelength was changed from 260 to $360 \mathrm{~nm}$ (Supplementary Fig. 26 and Table 1). This blue-shifted emission should be due to the gradually decreased intensities of the CPOA emission bands originating from 470 to $550 \mathrm{~nm}$ as the excitation wavelength varied from 260 to $360 \mathrm{~nm}$. To prove this phenomenon, the SSPL spectra at $77 \mathrm{~K}$ of $(\mathrm{R}, \mathrm{R})$-DAACH powder were also tested. As shown in Supplementary Fig. 27, because the nonradiative transition of triplet emission is greatly inhibited under freezing conditions, much-enhanced CPOA emissions were observed at $77 \mathrm{~K}$ compared to those under room temperature; and much obviously blue-shifted emissions from green to blue were observed in $(\mathrm{R}, \mathrm{R})$-DAACH powder, suggesting again that the abnormal hypochromatic shifted emission obtained under the excitation turning from 260 to $360 \mathrm{~nm}$ should be attributed to the decreased intensities of CPOA emissions.

Mechanism insights of CPOA. To seek the underlying origination of these unique optical properties, a further set of experimental and theoretical investigations including the SSPL in solutions, single-crystal analyses, and time-dependent density functional theory calculations on the $(\mathrm{R}, \mathrm{R})-\mathrm{DAACH}$ were conducted $^{36}$. As shown in Supplementary Figs. 28, 29, the excitation-dependent emissions were also observed in SSPL spectra of $(\mathrm{R}, \mathrm{R})-\mathrm{DAACH}$ in ethanol solutions with varied 

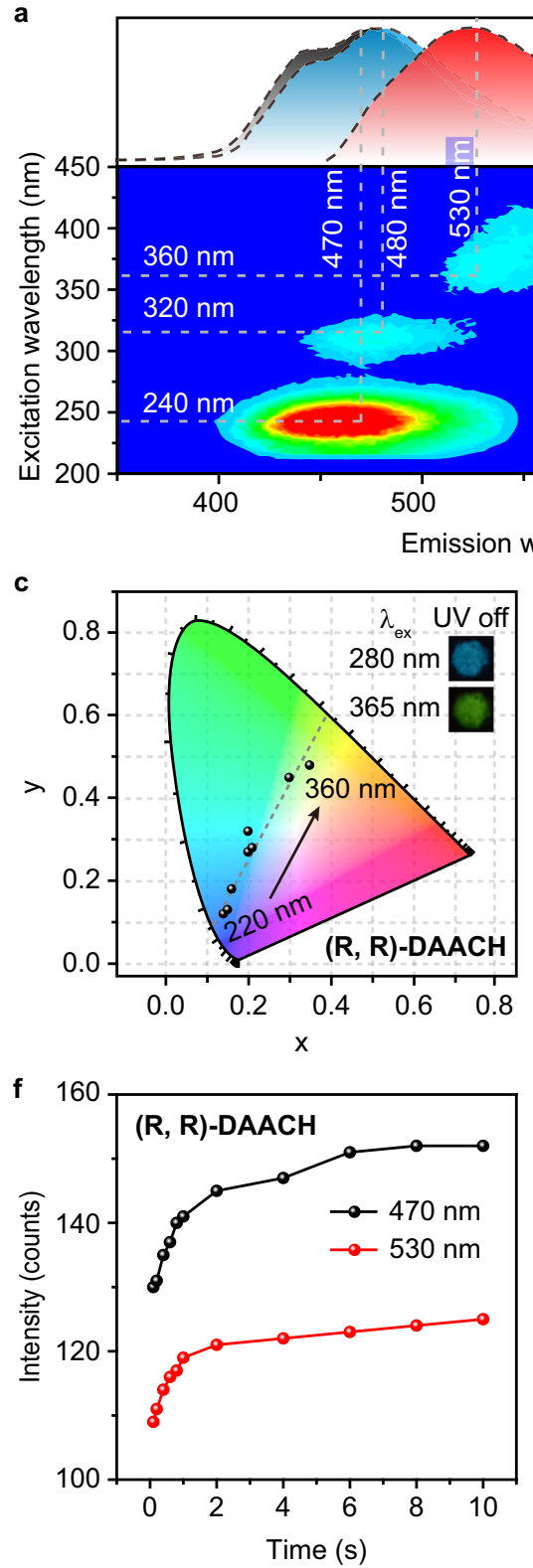

$\lambda$

$240 \mathrm{~nm}$

$320 \mathrm{~nm}$

- $360 \mathrm{~nm}$

(R, R)-DAACH
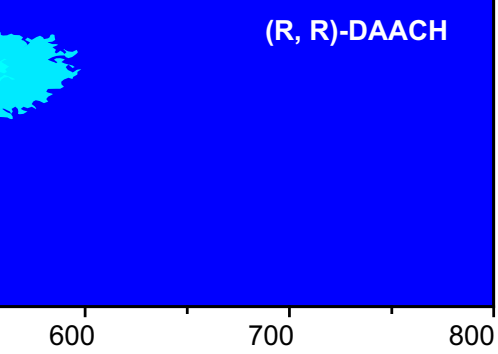

600

700

800

velength $(\mathrm{nm})$
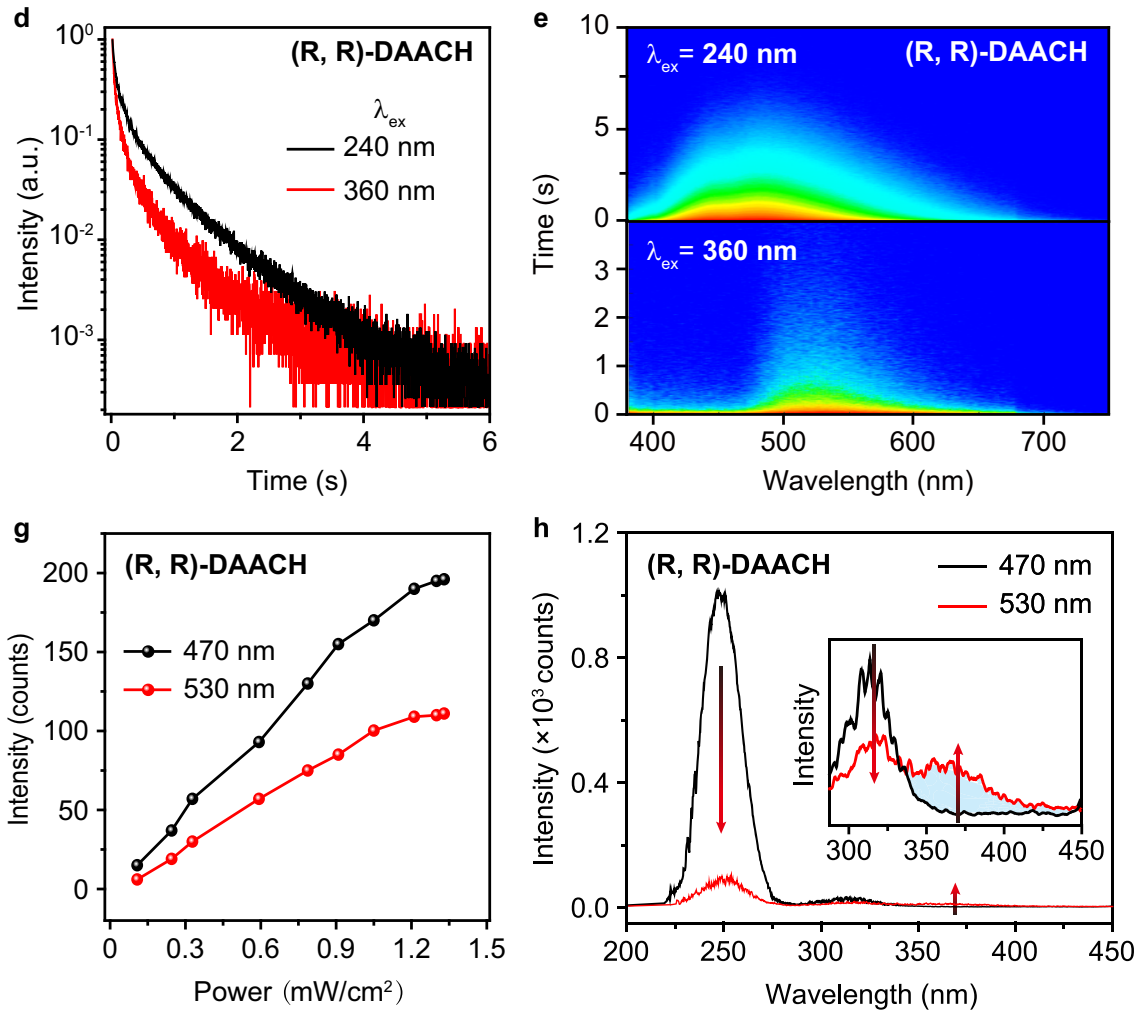

Fig. 2 Photophysical properties of the single-component color-tunable CPOA materials under ambient conditions. a Excitation-phosphorescence mapping of $(R, R)-D A A C H$ powder. The upper panel shows the CPOA spectra of ( $R, R)-D A A C H$ powder under excitation by 240,320 , and $360 \mathrm{~nm}$, respectively. $\mathbf{b}$ CPL properties of $(R, R)-D A A C H$ and $(S, S)$-DAACH powders under excitation by 240, 340, and 360 nm, respectively. c CIE coordinates variations of color-tunable CPOA spectra of $(\mathrm{R}, \mathrm{R})$-DAACH powder upon excitation by a different wavelength ranging from 220 to $360 \mathrm{~nm}$ with a step of $20 \mathrm{~nm}$. The insets exhibit the CPOA photographs of (R, R)-DAACH powder taken after turning off 280 and $365 \mathrm{~nm}$ excitation light, respectively. $\mathbf{d}$ Decay profiles of CPOA emission of (R, R)-DAACH powder upon excitation by 240 and $360 \mathrm{~nm}$, respectively. e Transient emission decay images of (R, R)DAACH powder following excitation by 240 (top) and $360 \mathrm{~nm}$ (bottom), respectively. $\mathbf{f}, \mathbf{g}$ CPOA intensity changes of (R, R)-DAACH powder at 470 and $530 \mathrm{~nm}$ as a function of irradiation time (f) and intensity ( $(\mathbf{g})$ upon excitation by 240 (black line) and $360 \mathrm{~nm}$ (red line), respectively. $\mathbf{h}$ Excitation spectra of $(\mathrm{R}, \mathrm{R})-\mathrm{DAACH}$ powder by monitoring CPOA peaks of 470 and $530 \mathrm{~nm}$, respectively.

concentrations upon excitation at different wavelengths under ambient conditions, which are consistent with the variation trend of (R, R)-DAACH powder (Supplementary Fig. 26), verifying again the presence of multiple emissive species as further revealed by varied fluorescence lifetimes and excitation-dependent cryogenic phosphorescence spectra under the excitation by different wavelengths (Supplementary Figs. 30-31). Notably, as the concentration increased from $10^{-5}$ to $10^{-2} \mathrm{M}$, the well-resolved peaks in the range of $400-500 \mathrm{~nm}$ exhibited largely enhanced intensities with slightly decreased lifetimes (Supplementary Fig. 32) and the red-shifted absorption spectra were found
(Supplementary Fig. 33). This high concentration enhanced luminescent intensities and red-shifted absorption bands of these unconventional luminophores should be ascribed to the enhanced molecular $p-\pi$ conjugation and intense intermolecular interactions in the aggregated state, thus leading to the efficient electron delocalization on diverse emissive centers via TSC for the formation of effective $\mathrm{CTE}^{34,37}$.

Crystallography analyses. To reveal the presence of different clusters in CPOA molecules, the intermolecular interactions and 

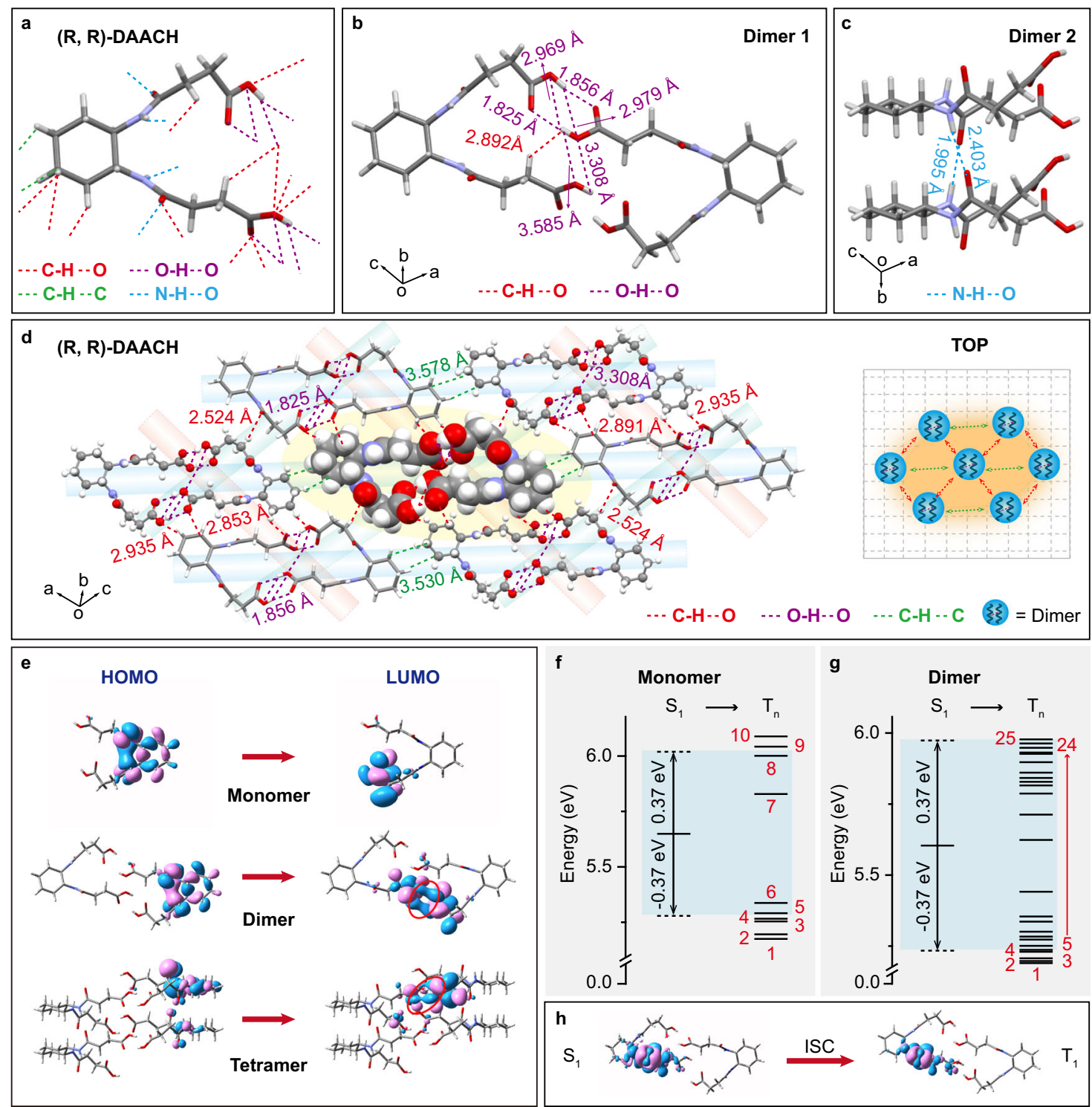

Fig. 3 Single crystal and theoretical analyses of color-tunable CPOA materials. a-d Intermolecular interactions of monomer (a), dimer (b, c), and molecular packing (d) in (R, R)-DAACH single crystal. The central dimer (highlighted by space fill mode) is stabilized by six identical dimers via efficient intermolecular interactions through neighbor dimers, thus leading to the diversified emission species with different cluster sizes. e Frontier molecular orbital distributions for the selected monomer, dimer, and tetramer in (R, R)-DAACH single crystal. $\mathbf{f}, \mathbf{g}$ TD-DFT calculated energy level diagram of selected monomer (f) and dimer $(\mathbf{g})$ in $(\mathrm{R}, \mathrm{R})$-DAACH single crystal. $\mathbf{h}$ Electron density difference in the singlet and triplet excited state of the dimer.

packing modes in the single crystal were carefully analyzed. (R, $\mathrm{R})$-DAACH adopted hairpin-shaped conformation in crystal (Fig. 3a), showing multiple effective intra/intermolecular interactions including $\mathrm{C}-\mathrm{H} \bullet \bullet \mathrm{O}, \mathrm{O}-\mathrm{H} \bullet \bullet \bullet \mathrm{O}, \mathrm{C}-\mathrm{H} \bullet \bullet \mathrm{C}$, and $\mathrm{N}-\mathrm{H} \bullet \bullet \bullet \mathrm{O}$. And obvious intermolecular interactions of $\mathrm{O}-\mathrm{H} \bullet \bullet \mathrm{O}$ with distances ranging from 1.825 to $3.585 \AA$, C-H...O with a distance of $2.892 \AA$, C-H $\bullet . . \mathrm{C}$ with distances of 3.530 and $3.578 \AA$, and $\mathrm{N}$ H...O with distances of 1.995 and $2.403 \AA$ were observed in different dimers and multimers (Fig. $3 b, c$ and Supplementary Figs. 34,35 ), which could not only promote the formation of rigid molecular configuration for suppressing non-radiative transitions but also facilitate TSC among different molecules ${ }^{38,39}$. These intense intermolecular interactions were also theoretically identified by the independent gradient model (IGM) analysis ${ }^{40}$, exhibiting a large IGM isosurface between adjacent molecules in both dimers, trimers, and tetramers (Supplementary Fig. 36). Extendedly, (R, R)-DAACH exhibited various interlocked dimers and multimers in the entire crystal (Fig. $3 c$ and Supplementary
Fig. 34) by utilizing the efficient intra/intermolecular interactions in neighboring dimers; and the central dimer was bonded by adjacent six dimers (Fig. 3d) in the same plane and these bonded dimers were further stabilized through intensive interlayer intermolecular interactions of N-H••O (Supplementary Fig. 35). These confinements in dimers could gift the different emission species with regulated and enhanced TSC for color-tunable CPOA that responds to varied excitation wavelength, probably leading to the central dimer for sky-blue afterglow, bonded dimers for green, and extendedly interlocked dimers for yellowish-green afterglows ${ }^{34,40}$.

Theoretical investigations. To further manifest our conceptions, we performed the theoretical calculations of the monomer and selected aggregates in ( $\mathrm{R}, \mathrm{R})-\mathrm{DAACH}$ single crystal. From the simulation results, the highest occupied molecular orbital (HOMO) and the lowest unoccupied molecular orbital (LUMO) 
(Fig. 3e) were separately localized on cyclohexane and acid arms in monomer; importantly, the HOMOs and LUMOs are remarkably delocalized on the cyclohexane and acid arms among different monomers for efficient through-space electron delocalization in the aggregated state. Furthermore, with the increment of the aggregate size, more obvious through-space delocalization and decreased energy bandgaps were observed (Supplementary Fig. 37). This kind of spatial frontier molecular orbital delocalization can not only narrow the energy bandgap but also lead to different clusters with plentiful triplet energy levels; these findings can be experimentally revealed by the red-shifted emission bands upon tuning the excitation wavelength from 240 to $360 \mathrm{~nm}$ and theoretically verified by the gradually decreased excited energy levels with the increasing of the aggregated size of a cluster. Moreover, to theoretically investigate the effective ISC in the aggregated state, the calculations of time-dependent density functional theory (TD-DFT) were carried out ${ }^{32,41}$. From the simulated excited state energy levels, there are only five triplet states showing singlet and triplet splitting energy $\left(\Delta E_{\mathrm{ST}}\right)$ lower than $0.37 \mathrm{eV}$ for enabling possible ISC in (R, R)-DAACH monomer according to the energy gap law (Fig. 3f and Supplementary Table 3$)^{23}$. In contrast, the total number of plausible ISC channels in the aggregated state was significantly increased to 20 for dimer, 30 for trimer, and 37 for tetramer (Fig. 3g, Supplementary Fig. 38, and Tables 4-8), respectively, thus facilitating high-efficiency ISC for the promotion of triplet excitons (Fig. 3h). Accordingly, on the basis of the experimental and theoretical investigations, we reason that the strong intra/intermolecular interactions in the same plane and interlayer should be responsible for the formation of different clusters to render efficient TSC for achieving varied emission species with tunable excited energy levels; on account of boosted ISC in the aggregated state, the photo-motivated singlet excitons can be facilely transformed to

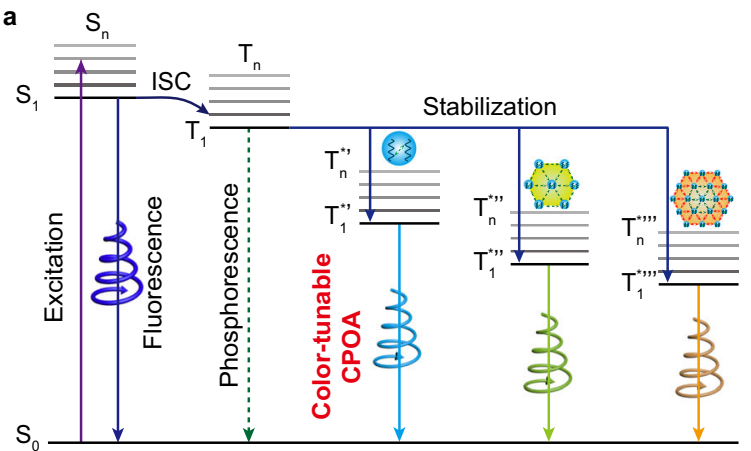

c

Color-tunable CPOA display
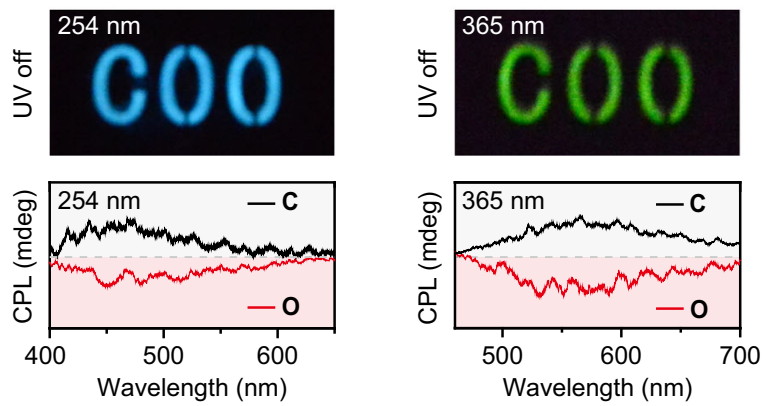

triplet excitons; taken together, on account of different emission species and high ISC rate in the aggregated state, excitationdependent color-tunable CPOA can be successfully achieved in this unconventional chirality cluster crystal (Fig. 4a).

Universality of the strategy. To testify the universality of this chiral clusterization strategy in constructing single-component color-tunable CPOA materials, other two pairs of enantiomers of $(\mathrm{R}, \mathrm{R}) /(\mathrm{S}, \mathrm{S})-\mathrm{DAPCH}$ and $\mathrm{L} / \mathrm{D}$-serine were explored. Indeed, $(\mathrm{R}, \mathrm{R}) /(\mathrm{S}, \mathrm{S})$-DAPCH demonstrate superb excitation-dependent CPOA properties, showing tunable emission color ranging from 410 to $530 \mathrm{~nm}$ (Supplementary Fig. 39). And, the singlecomponent color-tunable CPOA was also achieved in L/D-serine, exhibiting tunable chiral afterglow emissions spanning from deep blue to green (Supplementary Fig. 40).

Applications of the CPOA molecule. Benefiting from the excellent color-tunable CPOA emission, we take one step further to indicate the feasibility of (R, R)-DAACH and (S, S)-DAACH powders in fabricating multicolor CPL displays and visual UV color chart under ambient conditions (Fig. 4b) ${ }^{35}$. As designed, the "C" and "OO" characters in "COO" pattern were filled with (S, S)-DAACH and (R, R)-DAACH powders, respectively. With the excitation wavelength changing from 254 to $365 \mathrm{~nm}$, the OA images of "COO" were correspondingly changed from blue to yellowish-green, demonstrating the success in achieving multicolor CPOA displays (Fig. 4b, c, top). More interestingly, because of the outstanding CPL feature of (S, S)-DAACH and (R, R)DAACH powders, the multicolor CPL displays were facilely obtained (black for positive CPL and red for negative CPL) (Fig. 4c, bottom). More strikingly, since the color-tunable CPOA images upon excitation by invisible UV light spanning from 240 to $360 \mathrm{~nm}$ with a step of $40 \mathrm{~nm}$ can be easily recognized by the

b

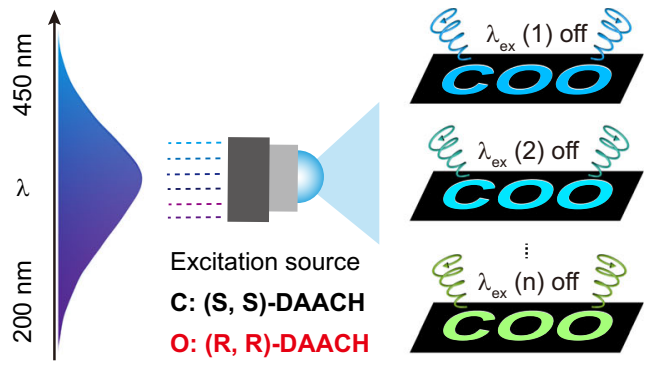

d

UV color chart

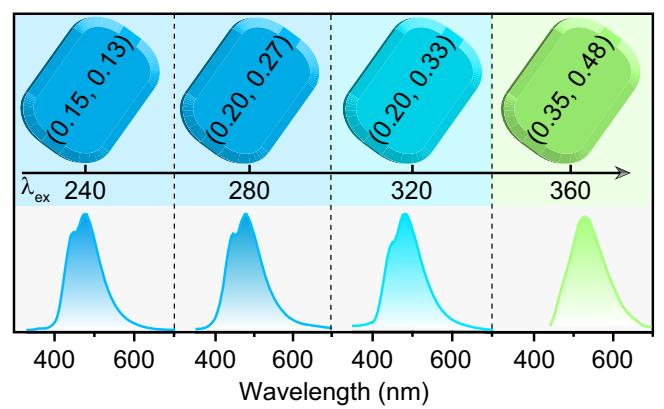

Fig. 4 Mechanism and applications of the color-tunable CPOA molecules. a Plausible energy transfer processes for color-tunable CPOA. b Schematic drawing of multicolor CPOA displays by varying the excitation wavelength of UV light. c Color-tunable CPOA display of COO pattern using (S, S)-DAACH to fabricate " $\mathrm{C}$ " and (R, R)-DAACH for "OO" and the corresponding CPL curves of " $\mathrm{C}$ " (black) and " $\mathrm{O}$ " (red) in the chiral-featured pattern upon 254 (left) and 365 (right) nm UV light excitation, respectively. $\mathbf{d}$ Excitation-dependent color chart of CPOA spectra with the corresponding CIE values demonstrating the possibility of $(R, R)-D A A C H$ powder in serving as sensing medium for visual RTP detection of particular UV light wavelengths. 
naked eyes, these chiral cluster crystals may hold substantial promise for advanced visualization of UV sensing (Fig. 4d).

\section{Discussion}

In summary, we have succeeded in achieving color-tunable CPOA from a single-component chiral cluster crystal through chiral clusterization. The direct linkage between the chirality center of trans-1, 2-diamidocyclohexane, and succinic acid containing multiple heteroatoms can not only yield efficient CPL emission and diverse CTE emission species but also boost efficient ISC and strong non-covalent intermolecular interactions to promote the generation of triplet excitons and suppression of non-radiative decay for efficient RTP. More importantly, owing to the spectacular chiral clusterization effects, dynamically selective response to the excitation wavelength for color-tunable CPOA emission was observed in the single-component molecular clusters. With the changing of excitation wavelength from 240 to $360 \mathrm{~nm}$, the two enantiomers of (S, S)-DAACH and (R, R)-DAACH exhibited excellent color-tunable CPOA spanning from blue $(\sim 470 \mathrm{~nm})$ to yellowish-green $(\sim 530 \mathrm{~nm})$ with $\left|\mathrm{g}_{\text {lum }}\right|$ values higher than $2.3 \times 10^{-3}$ and lifetime up to $587 \mathrm{~ms}$. Taking advantage of the spectacular excitation-dependent color-tunable CPOA molecules, multicolor CPL displays and visual UV color charts were successfully constructed. The realization of multicolor-tunable CPOA emission from a single-component chiral cluster crystal through a one-stone-two-birds approach could afford fundamental and vital design clues for the exploration of smart multicolor CPL and RTP functional materials, shedding important light on the construction of multifunctional and high-performance new-concept organic molecules for advanced optoelectronic applications.

\section{Methods}

Unless otherwise noted, all reagents were purchased from Aldrich and Acros and used without additional purification. The molecular structures of the assynthesized phosphorescent molecules were characterized by single-crystal X-ray diffraction, NMR spectroscopy, HRMS, and MALDI-TOF MS (see the Supplementary Information).

The steady-state UV absorption data were collected on a Jasco V-750 spectrophotometer. The intrinsic circularly polarized luminescence (CPL) spectra were investigated using a JASCO CPL-300 spectrometer. The circular dichroism (CD) spectra were measured on a JASCO J-810 circular dichroism spectrometer. The fluorescence spectra, phosphorescence spectra, kinetic measurements, lifetimes of the room-temperature phosphorescence (RTP) were measured using an Edinburgh FLS980 fluorescence spectrophotometer equipped with a xenon arc lamp (Xe900), laser, and a microsecond ( $\mu$ s) flash-lamp (uF900), respectively. All the data of these single-crystal structures were collected on a Bruker SMART APEX (II)-CCD at $100 \mathrm{~K}$. The photos and supporting videos were recorded by Nikon D90. The TDDFT calculations were performed with the Gaussian 09 program. All the computational models were built from single-crystal structures without further geometry optimization. The excitation energy of the $n$th singlet state $\left(\mathrm{S}_{n}\right)$ and the $n$-th triplet state $\left(T_{n}\right)$ states were calculated at the TD-DFT method of B3LYP/6-31 G(d,p) level based on the monomer and selected aggregates extracted from the single-crystal. Frontier molecular orbital distributions were calculated based on the monomer and selected aggregates extracted from the single-crystal at M06-2X/cc-pVTZ.

\section{Data availability}

The data that support the findings of this study are available from the corresponding author upon request. Crystallographic data generated in this study for (R,R)-DAACH has been deposited in The Cambridge Crystallographic Data Centre under accession code CCDC 2083718 .

Received: 10 August 2021; Accepted: 24 December 2021;

Published online: 20 January 2022

\section{References}

1. MacKenzie, L. E., Pålsson, L., Parker, D., Beeby, A. \& Pal, R. Rapid timeresolved circular polarization luminescence (CPL) emission spectroscopy. Nat. Commun. 11, 1676 (2020).
2. Neugebauer, M., Banzer, P. \& Nechayev, S. Emission of circularly polarized light by a linear dipole. Sci. Adv. 5, v7588 (2019).

3. MacKenzie, L. E. \& Pal, R. Circularly polarized lanthanide luminescence for advanced security inks. Nat. Rev. Chem. 5, 109-124 (2020).

4. Ayuso, D. et al. Synthetic chiral light for efficient control of chiral light-matter interaction. Nat. Photonics. 13, 866-871 (2019).

5. Han, D. et al. Sequentially amplified circularly polarized ultraviolet luminescence for enantioselective photopolymerization. Nat. Commun. 11, 5659 (2020).

6. $\mathrm{Wu}, \mathrm{X}$. et al. Exploiting racemism enhanced organic room-temperature phosphorescence to demonstrate Wallach's rule in the lighting chiral chromophores. Nat. Commun. 11, 2145 (2020).

7. Zheng, H. et al. Uncovering the circular polarization potential of chiral photonic cellulose films for photonic applications. Adv. Mater. 30, 1705948 (2018).

8. Li, J., Bisoyi, H. K., Tian, J., Guo, J. \& Li, Q. Optically rewritable transparent liquid crystal displays enabled by light-driven chiral fluorescent molecular switches. Adv. Mater. 31, 1807751 (2019).

9. Hu, M., Feng, H., Yuan, Y., Zheng, Y. \& Tang, B. Z. Chiral AIEgens-chiral recognition, CPL materials and other chiral applications. Coordin. Chem. Rev. 416, 213329 (2020)

10. Zhao, T. et al. Dual-mode induction of tunable circularly polarized luminescence from chiral metal-organic frameworks. Research. 2020, 1-12 (2020).

11. Ji, L. et al. Dimension-tunable circularly polarized luminescent nanoassemblies with emerging selective chirality and energy transfer. ACS Nano 14, 2373-2384 (2020)

12. Maeda, H. et al. Chemical-stimuli-controllable circularly polarized luminescence from anion-responsive $\pi$-conjugated molecules. J. Am. Chem. Soc. 133, 9266-9269 (2011).

13. Han, J., You, J., Li, X., Duan, P. \& Liu, M. Full-color tunable circularly polarized luminescent nanoassemblies of achiral aiegens in confined chiral nanotubes. Adv. Mater. 29, 1606503 (2017).

14. Zhao, B., Pan, K. \& Deng, J. Combining chiral helical polymer with achiral luminophores for generating full-color, on-off, and switchable circularly polarized luminescence. Macromolecules. 52, 376-384 (2018).

15. Nishikawa, T., Nagata, Y. \& Suginome, M. Poly(quinoxaline-2,3-diyl) as a multifunctional chiral scaffold for circularly polarized luminescent materials: color tuning, energy transfer, and switching of the cpl handedness. ACS Macro Lett. 6, 431-435 (2017).

16. Albano, G., Pescitelli, G. \& Di Bari, L. Chiroptical properties in thin films of $\pi$ conjugated systems. Chem. Rev. 120, 10145-10243 (2020).

17. Xu, M. et al. Designing hybrid chiral photonic films with circularly polarized room-temperature phosphorescence. Acs Nano 14, 11130-11139 (2020).

18. Liu, R., Ding, B., Liu, D. \& Ma, X. Switchable circularly polarized roomtemperature phosphorescence based on pure organic amorphous binaphthyl polymer. Chem. Eng. J. 421, 129732 (2021).

19. Liang, X. et al. Organic room-temperature phosphorescence with strong circularly polarized luminescence based on paracyclophanes. Angew. Chem. Int. Ed. 58, 17220-17225 (2019).

20. Li, J. et al. Colour-tunable dual-mode afterglows and helical-array-induced mechanoluminescence from AIE enantiomers: effects of molecular arrangement on formation and decay of excited states. Chem. Eng. J. 418, 129167 (2021).

21. Hirata, S. \& Vacha, M. Circularly polarized persistent room-temperature phosphorescence from metal-free chiral aromatics in air. J. Phys. Chem. Lett. 7, 1539-1545 (2016).

22. Chen, W. et al. Long-persistent circularly polarized phosphorescence from chiral organic ionic crystals. Chem. Eur. J. 24, 17444-17448 (2018).

23. $\mathrm{Li}, \mathrm{H}$. et al. Stimuli-responsive circularly polarized organic ultralong room temperature phosphorescence. Angew. Chem. Int. Ed. 59, 4756-4762 (2020).

24. Favereau, L. et al. Persistent organic room-temperature phosphorescence in cyclohexane-trans-1,2-bisphthalimide derivatives: the dramatic impact of heterochiral vs homochiral interactions. J. Phys. Chem. Lett. 11, 6426-6434 (2020).

25. Zhou, Q. et al. Clustering-triggered emission of nonconjugated polyacrylonitrile. Small. 12, 6586-6592 (2016).

26. Wang, Y. et al. Nonconventional luminophores with unprecedented efficiencies and color-tunable afterglows. Mater. Horiz. 7, 2105-2112 (2020).

27. Dou, X. et al. Color-tunable, excitation-dependent, and time-dependent afterglows from pure organic amorphous polymers. Adv. Mater. 32, 2004768 (2020).

28. Yang, J. et al. The influence of the molecular packing on the room temperature phosphorescence of purely organic luminogens. Nat. Commun. 9, 840 (2018).

29. Yang, Z. et al. Boosting the quantum efficiency of ultralong organic phosphorescence up to $52 \%$ via intramolecular halogen bonding. Angew. Chem. Int. Ed. 59, 17451-17455 (2020).

30. Ruiz-Carretero, A. et al. Stepwise self-assembly to improve solar cell morphology. J. Mater. Chem. A. 1, 11674-11681 (2013). 
31. Li, H. et al. Fluorine-induced aggregate-interlocking for color-tunable organic afterglow with a simultaneously improved efficiency and lifetime. Chem. Sci. 12, 3580-3586 (2021).

32. Ma, H., Peng, Q., An, Z., Huang, W. \& Shuai, Z. Efficient and long-lived room-temperature organic phosphorescence: theoretical descriptors for molecular designs. J. Am. Chem. Soc. 141, 1010-1015 (2019).

33. Zhao, W. et al. Rational molecular design for achieving persistent and efficient pure organic room-temperature phosphorescence. Chem 1, 592-602 (2016).

34. Zheng, S., Zhu, T., Wang, Y., Yang, T. \& Yuan, W. Z. Accessing tunable afterglows from highly twisted nonaromatic organic aiegens via effective through-space conjugation. Angew. Chem. Int. Ed. 59, 10018-10022 (2020).

35. Gu, L. et al. Colour-tunable ultra-long organic phosphorescence of a singlecomponent molecular crystal. Nat. Photonics. 13, 406-411 (2019).

36. Tang, L. et al. X-ray excited ultralong room-temperature phosphorescence for organic afterglow scintillators. Chem. Commun. 56, 13559-13562 (2020).

37. Zhang, $\mathrm{H}$. et al. Clusterization-triggered emission: uncommon luminescence from common materials. Mater. Today 32, 275-292 (2020).

38. Mao, Z. et al. Two-photon-excited ultralong organic room temperature phosphorescence by dual-channel triplet harvesting. Chem. Sci. 10, 7352-7357 (2019).

39. Xie, Y. et al. How the molecular packing affects the room temperature phosphorescence in pure organic compounds: ingenious molecular design, detailed crystal analysis, and rational theoretical calculations. Adv. Mater. 29, 1606829 (2017).

40. Chen, L. et al. Crystal structure characterization, independent gradient model analysis, and gas-phase-mediated transformation of nicosulfuron $\mathrm{dmf}$ solvate and hydrate. Cryst. Res. Technol. 54, 1800244 (2019).

41. Tao, Y. et al. Resonance-activated spin-flipping for efficient organic ultralong room-temperature phosphorescence. Adv. Mater. 30, 1803856 (2018).

\section{Acknowledgements}

This study was supported in part by the National Natural Science Foundation of China (62075102 awarded to HL, 22075149 awarded to YT, 21772095 awarded to CZ, 61875090, and 91833306 awarded to RC), the Jiangsu Specially-Appointed Professor Plan (awarded to YT), the Six Talent Plan of Jiangsu Province (XCL-049 awarded to YT), Key giant project of Jiangsu Educational Committee (19KJA180005 awarded to RC), the fifth 333 project of Jiangsu Province of China (BRA2019080 awarded to RC), 1311 Talents Program of Nanjing University of Posts and Telecommunications (Dingshan awarded to YT), Natural Science Fund for Colleges and Universities in Jiangsu Province (20KJB430001 awarded to HL), China Postdoctoral Science Foundation-funded project (2018M642284 awarded to HL), and Nanjing University of Posts and Telecommunications Start-up Fund (NUPTSF) (NY220151 awarded to GX, NY219007 awarded to YT, NY219160 awarded to PL, and NY217140 awarded to HL).

\section{Author contributions}

HL, YT, WH, and RC conceived and designed the experiments. HL, JW, HL, and GX measured and analyzed the photophysical properties. ZW, CZ, and PL performed the computational calculations. HL, JG, and FH fabricated the applications. HL, YT, WH, and RC wrote the manuscript and all authors contributed to the data analysis.

\section{Competing interests}

The authors declare no competing interests.

\section{Additional information}

Supplementary information The online version contains supplementary material available at https://doi.org/10.1038/s41467-022-28070-9.

Correspondence and requests for materials should be addressed to Ye Tao, Wei Huang or Runfeng Chen.

Peer review information Nature Communications thanks Peng $\mathrm{Wu}$, and the other, anonymous, reviewer(s) for their contribution to the peer review of this work. Peer reviewer reports are available.

Reprints and permission information is available at http://www.nature.com/reprints

Publisher's note Springer Nature remains neutral with regard to jurisdictional claims in published maps and institutional affiliations.

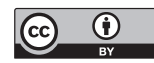

Open Access This article is licensed under a Creative Commons Attribution 4.0 International License, which permits use, sharing, adaptation, distribution and reproduction in any medium or format, as long as you give appropriate credit to the original author(s) and the source, provide a link to the Creative Commons license, and indicate if changes were made. The images or other third party material in this article are included in the article's Creative Commons license, unless indicated otherwise in a credit line to the material. If material is not included in the article's Creative Commons license and your intended use is not permitted by statutory regulation or exceeds the permitted use, you will need to obtain permission directly from the copyright holder. To view a copy of this license, visit http://creativecommons.org/ licenses/by/4.0/.

(C) The Author(s) 2022 